\title{
La ciencia económica en tiempos de crisis
}

\author{
Economic Science in Crisis Times
}

A ciência econômica em tempos de crise

William Orlando Prieto-Bustos *

Joan Miguel Tejedor-Estupiñán **

DOI: https://doi.org/10.19053/01203053.v36.n64.2017.5937

Fecha de recepción: 20 de marzo de 2017

Fecha de aprobación: 25 de mayo de 2017

* Magíster en Economía y economista. Docente de la Facultad de Ciencias Económicas y Administrativas de la Universidad Católica de Colombia. Bogotá Colombia. Código Postal: 111311. Correo electrónico: woprieto@ ucatolica.edu.co - (D) https://orcid.org/0000-0001-7992-8781

** PhD (c) in Economics, magíster en Derechos Humanos y economista. Docente de la Facultad de Ciencias Económicas y Administrativas de la Universidad Católica de Colombia. Bogotá, Colombia. Correo electrónico: jmtejedor@ucatólica.edu.co - iD https://orcid.org/0000-0002-2346-3222 


\section{Resumen}

El presente documento discute la importancia de los principios de pluralismo crítico, responsabilidad, primun non nocere y precaución, en un esquema de reflexión ética de la economía como ciencia y como técnica. Para tal efecto se realiza un análisis institucional de políticas, normas y directrices en una muestra de diez países: ocho de Latinoamérica, más Estados Unidos e Inglaterra. El principal resultado indica que el actual régimen institucional tanto legislativo como ético no soluciona la tensión que genera el interés privado y el interés público en el desempeño profesional de un economista en una economía de mercado. Es necesario incorporar una articulación flexible que facilite una interpretación sencilla y concreta que oriente al mejoramiento de la calidad del servicio privado y público que desempeña un economista.

Palabras clave: enfoque científico, conocimiento científico, saber científico.

Clasificación JEL: A11, A13, A14. 


\section{Abstract}

This paper discusses the importance of critical pluralism, responsibility, primun non nocere and caution principles, in a scheme of ethical reflection of economics as a science and as a technique. For this purpose, an institutional analysis of policies, standards and guidelines is carried out in a sample of 10 countries: 8 from Latin America, along with the United States, and England. The main result indicates that the current institutional regime, both legislative and ethical, does not solve the tension generated by private interest and public interest in the professional performance of an economist in a market economy. It is necessary to incorporate a flexible articulation that facilitates a simple and concrete interpretation that guides the improvement of the quality of the private and public service that an economist plays.

Keywords: scientific approach, scientific knowledge, case studies. 


\section{Resumo}

Este artigo discute a importância dos princípios do pluralismo crítico, prestação de contas, primum non nocere e cautela, em um contexto de reflexão ética da economia como ciência e técnica oito na América Latina, além de Estados Unidos e Inglaterra para esta finalidade uma análise institucional de políticas, normas e diretrizes em uma amostra de dez países é realizada. O principal resultado indica que os actuais acordos institucionais legislativas e éticas não resolve a tensão gerada pelo interesse privado eo interesse público no desempenho profissional de um economista em uma economia de mercado. É necessário incorporar uma articulação flexível que facilita uma interpretação simples e concreto para orientar a melhoria da qualidade do serviço público e peças privadas economista.

Palavras-chave: abordagem científica, o conhecimento científico, o conhecimento científico. 
Apuntes CENES Volumen 36, Número 64

julio - diciembre 2017. Págs. 47-78

\section{INTRODUCCIÓN}

La economía parece estar ad portas de una revolución científica que requiere de un cambio del actual paradigma que orienta la política económica. Lo anterior ha traído como consecuencia una tensión en el ejercicio profesional que intenta articular un interés privado que define sus resultados, con un interés público que define su credibilidad. Dichas tensiones han ocasionado fallas sistemáticas en la profesión, que están modificando la relación entre el sujeto y el objeto de la ciencia económica, y entre el sujeto y los procedimientos a través de los cuales estudia el objeto en condiciones de incertidumbre.

El presente documento analiza políticas, normas y directrices institucionales de la profesión de economista para una muestra de diez países: Colombia, Venezuela, Ecuador, Perú, Argentina, México, Costa Rica, Estados Unidos e Inglaterra. La discusión se presenta en torno al cambio de paradigma científico en la economía posterior a la crisis financiera de 2007, sugerido por autores como Galbraith (2010), Stiglitz (2008), Buiter (2009), Kaletsky (2009), Colander et al. (2009), Krugman (2009), Freeman (2009), Mayer (2009), DeMartino (2005), y Herman y Flecker (2003). En particular, se discute críticamente el ejercicio de la profesión desde las tensiones que sugiere la importancia de la economía en el orden institucional que afecta la distribución y la producción de bienes y servicios y el interés público que suscitan los resultados de diferentes órdenes económicos sobre el bienestar de la sociedad. 
El documento está dividido en cuatro secciones; en la primera se presenta la relación entre prestigio y credibilidad de la profesión e interés público, a partir de las definiciones de la profesión propuestas por la Organización Internación del Trabajo (O.I.T.). En la segunda sección se discute la regulación que presupone la confusión del interés público y el interés privado, implementando una metodología de análisis comparativo a través de un contraste analítico de las regulaciones y códigos de comportamiento profesional de los diez países que conforman la muestra por conveniencia propuesta de estudio. La muestra fue seleccionada sobre los desarrollos en ética de la economía, y la importancia de la definición de un enfoque ético en la configuración de un paradigma tanto teórico como aplicado de una economía del desarrollo en América Latina. La tercera sección propone una discusión de los resultados del análisis comparativo, a partir del principio de racionalidad económica que sostiene el diseño de políticas públicas para corregir la falla de mercado que ocasiona la información incompleta acerca de la calidad de los servicios profesionales de los economistas. Finalmente se presentan las principales conclusiones.

CREDIBILIDAD DE LA PROFESIÓN E INTERÉS PÚBLICO

Nunca antes el conocimiento había experimentado una expansión tan significativa como en las sociedades posfordianas de la información. El conocimiento, ligado estrechamente al surgimiento de habilidades y destrezas en el quehacer humano, es el núcleo constitutivo per se de una profesión fundada sobre un ejercicio científi-co. De acuerdo con Bejarano (1999), quien define una profesión como la aplicación práctica y rutinaria de un conocimiento, se hace necesario un análisis de los componentes sociológicos que se estructuran en torno al contexto del ejercicio profesional, el prestigio y la influencia de la profesión en la sociedad. En tal definición de una profesión sobresalen dos características. En primera instancia, la existencia de un monopolio sobre un contenido de conocimiento formal, susceptible de ser aplicado en la práctica. En segunda instancia, la ocurrencia de un monopolio sobre un mercado de servicios profesionales relacionados.

Teniendo en cuenta lo anterior, desde la perspectiva sociológica, el proceso de formalización de una profesión, según Bejarano (1999), puede describirse en tres fases. En la primera fase ocurren aprendizajes formales e informales. En la segunda y como consecuencia de la anterior aparecen formas organizativas tales como las organizaciones de enseñanza y organizaciones de agremiación de profesionales que controlan el acceso a la profesión con la correspondiente 
estandarización, y la emisión de licencias para ejercer en términos del uso teórico y práctico del conocimiento especializado. En la tercera fase aparecen condiciones legales del desempeño laboral a partir de una evaluación del impacto de la monopolización del ejercicio profesional sobre la acción profesional. En tal sentido, el prestigio social de la profesión per se y en relación con el mercado laboral representa un correlato del desarrollo de las tres fases de la profesionalización.

La actividad profesional supone la interrelación entre el sujeto que posee el conocimiento y un objeto a través del cual se expresa el conocimiento que otorga la profesión. Superpuesta en un marco social, la definición de profesión está sujeta a una responsabilidad social que supera el interés privado que la origina, y establece su prestigio y credibilidad. Ogus (1994) describe la importancia de la integridad profesional ${ }^{1}$ en la formación de prestigio y credibilidad al definir una profesión como una ocupación que requiere especialización de habilidades y destrezas, las cuales son adquiridas a través de entrenamiento intelectual, cuya prestación de servicios se realiza con alto grado de integridad y se rige por una relación de contraprestación con un usuario.

Como profesión, la economía estudia las acciones desarrolladas por el ser humano para satisfacer necesidades con un uso racional de recursos escasos. Por lo tanto, el conocimiento especializado surge como consecuencia de la observación directa de los procesos históricos, desde una perspectiva materialista de la producción, la distribución y el consumo. El más alto nivel de capacitación ocurre a partir de la formación en teoría económica que se sustenta mediante la aplicación de un método cientí ico ${ }^{2}$ basado en la observación, la descripción, la formulación de hipótesis, la demostración o la comparación de las hipótesis, y la comparación universal. Debido a que "su materia prima está ligada a intereses e ideologías" (Allais, 1978, p. 24.), la economía no es considerada una ciencia formal. A diferencia de las matemáticas en donde la demostración equivale a la experimentación, la construcción de conocimiento económico requiere del examen de la evidencia empírica para considerarse materia de la profesión.

\footnotetext{
${ }^{1}$ Desde el punto de vista ético, sería la manera de manejarse coherentemente con los valores personales y compartidos con la comunidad a la que se pertenece.

2 Proceso científico criticado con vehemencia a partir de la crisis financiera de 2008. Ver Krugman, P. (2009), Kaletsky, A. (2009), Freeman, A. (2009), Buiter, W. (2009), Cohen, P (2007), Colander, D. et. al. (2009), Stiglitz, J. (2008), y Galbraith J. (2010).
} 
La etimología del término economía hace referencia a la actividad relacionada con la administración de un recurso privado. Los griegos denominaban oikos a la casa habitada por un administrador nemo, cuya capacidad administrativa de los recursos privados dio origen a la actividad económica de la cual se deriva la palabra economía. Desde 1615, cuando Antonio de Montchretien a irmó que la ciencia de la administración del recurso privado era la ciencia de la administración del recurso público, en cuanto el Estado, al igual que la familia, administraba una riqueza colectiva en un espacio común, la economía incorporó el adjetivo política para extender el signi icado al ámbito público. Posteriormente, con la aparición en 1776 de la Riqueza de las Naciones, obra cumbre de Adam Smith (1776), la economía inicia un proceso orientado a la explicación de la escasez y la acumulación material que persiste hasta nuestros días.

Cuando Bejarano (1999) describe los antecedentes de la profesión de economista, destaca aspectos institucionales internacionales y nacionales en la formalización de la profesión en el mundo y en Colombia. En el ámbito internacional sobresale la aparición de las escuelas de economía en 1895 , posterior a la aparición de las primeras agremiaciones de economistas en 1795. Mientras tanto, en Colombia, la fase preformal y la fase de enseñanza formal fueron subsidiarias de otras ramas de formación académica, la profesionalización tuvo un alto componente autodidacta, marcado por una fuerte relación con la ingeniería y el derecho. La institucionalización del conocimiento fue lograda gracias a la implementación de misiones tales como la misión Kemerer en 1930, la misión Currie de 1950, las misiones de la Cepal 1954 y 1958 y la misión Lebrett (1955), que permitieron la superación de la enseñanza informal, consolidando el proceso de profesionalización de la ciencia económica en Colombia.

En la actualidad, la dificultad más evidente de la profesión radica en la creciente importancia del interés público en la práctica del conocimiento, el cual se construye en el interior de la ciencia económica. La percepción de la sociedad con relación a la profesión, bien puede localizarse en dos extremos opuestos; en el primero, los economistas adquieren un rótulo de expertos que disponen de un conocimiento especializado que afecta la fortuna y la pobreza de la sociedad sobre la cual se dispone el bienestar social; en el segundo, los economistas son considerados un clan privilegiado y excluyente, poseedor de un conocimiento particular utilizado para priorizar el interés privado sobre el interés público ${ }^{3}$. La tensión de percepción de la opinión

\footnotetext{
${ }^{3}$ Samuel (2000), Bloom (2004) Garoupa (2004) y Stephen (2004) presentan análisis pormenorizados desde una perspectiva no técnica y técnica de los inconvenientes que genera el monopolio de conocimientos de las profesiones en el mejoramiento del bienestar social.
} 
pública en los dos extremos genera cuatro tipos de conflictos para la profesión ${ }^{4}$ del economista:

1. El público, en general, puede tener expectativas no razonables acerca de lo que puede o no alcanzar el análisis económico y la política económica en términos del bienestar individual y colectivo, debido a la existencia de incertidumbre e instituciones que median los arreglos económicos dispuestos en el mercado.

2. El público, en general, es excluido de la comprensión de los aportes y desventajas más relevantes de alternativas económicas competitivas, debido al desconocimiento de prácticas y conceptos de uso común en la teoría y práctica de la ciencia económica y la política económica.

3. El economista no considera un deber publico transmitir en forma precisa y comprensible el propósito, las dificultades, las restricciones y los resultados de su labor.

4. Cuando se trata de economistas que desempeñan funciones en cargos de interés público como las instituciones públicas y las organizaciones no gubernamentales, el público, en general, asume que la profesión debe priorizar el interés público sobre el interés privado, desconociendo la importancia de la ideología y los intereses económicos que surgen por la imposibilidad de separar el objeto de estudio, la sociedad, del sujeto que la estudia, el economista.

Lo anterior genera una tensión permanente, tanto en la aplicación de conocimientos como en el desempeño esperado de los economistas, quienes, en tiempos de revolución científica ${ }^{5}$ o cambio de paradigma referencial, no encuentran una explicación consistente y coherente que articule el interés público con el interés privado. Por lo anterior, dada la metodología de enseñanza de la teoría económica dominante, resulta mucho más fácil distinguir el interés privado del interés público desde la perspectiva de los profesionales en economía comparada con la perspectiva del público en general. Aun cuando la responsabilidad ética debería estar en la profesión, Bloom (2004) afirma que la educación profesional tiende a enfatizar la especialización que facilita la solución de problemas del contratista, con lo cual el mismo entrenamiento limita la sensibilidad profesional necesaria para contextualizar

\footnotetext{
${ }^{4}$ Bloom (2004) presenta cuatro posibles conflictos del interés público y el interés privado para las profesiones en general.

${ }^{5}$ Para algunos autores como Kaletsky (2009) y Freeman (2009), la crisis financier es eventualmente un rompimiento con el análisis de equilibrio de la doctrina dominante en economía.
} 
los efectos de la actividad profesional sobre el interés público. Hecho que es más evidente en la economía, como lo describe McCloskey (2009) al referirse a la economía de Samuelson que sugiere que la ciencia económica trata exclusivamente acerca del carácter utilitario del personaje "Max U", cuya alternativa de vida supone la avaricia como una alternativa factible ${ }^{6}$.

En un escenario de información incompleta $^{7}$, la tensión del interés privado y el interés público en el desempeño de las actividades concernientes a la profesión del economista, aumenta con la imposición de conductas profesionales que limitan la capacidad de movimiento del profesional ante conductas inapropiadas de sus superiores, y del principal agente de auditoría, la sociedad. Los acuerdos de confidencialidad y códigos de ética "sobre la información" se elaboran sin una concepción apropiada del interés público, lo que significa servir al interés público o como se llama en la actualidad, "el bien común", desde una clara perspectiva que privilegia el interés privado.

La ausencia de una concepción más precisa del interés público se hace más evidente en las definiciones que sobre la profesión propone la Organización Internacional del Trabajo (OIT). La falta de una actividad relacionada con la reflexión ética, tanto en la dimensión científica como en la dimensión política-técnica de la proesión, es común a ambas propuestas. Las definiciones y las tareas del economista se relacionan con un conjunto de técnicas, procedimientos, reglas, normas o protocolos que tienen como objetivo alcanzar un resultado sobre el cual ninguna de las funciones o tareas asignadas establece una reflexión ética (ver Anexo 1). Es decir, la reflexión crítica en el ámbito de la ética ausente de las funciones que caracterizan la profesión queda dispuesta en el ámbito transversal de las competencias del ser, generando una disyuntiva al enfrentar la separación del interés público y el interés privado por parte del economista al ofrecer servicios profesionales.

La OIT (2008) afirma que "los economistas realizan estudios o investigaciones con el objeto de desarrollar o perfeccionar conceptos, teorías, y métodos relativos al comportamiento de mercados..."; de donde se presume que la categoría economista está sujeta a la existencia de mercados,

\footnotetext{
${ }^{6}$ Una vez los banqueros en el sistema financiero asumen la existencia de Max $U$, justifican las estrategias de maximización de los precios de los activos con base en la hipótesis de mercados eficientes y expectativas racionales, como una manera óptima de articular el interés privado con el interés público, justo antes de la explosión de la burbuja financiero de los precios de los derivados en el mercado de las hipotecas en 2007

7 Asimétrica en el caso de los banqueros que tienen un conocimiento mayor acerca de los comportamientos de riesgo moral que subyacen a la crisis financiera
} 
cuya función es la de formular políticas económicas y prever soluciones para problemas económicos sin ninguna consideración normativa acerca del resultado que persiguen las diferentes técnicas o políticas económicas, con lo cual no es posible distinguir en dicha definición el interés público del interés privado.

Lo anterior refleja una orientación instrumental en la búsqueda de soluciones económicas sin consideración alguna a las consideraciones teóricas, sin justificación en una problemática definida, pero necesarias en la comprensión del funcionamiento de un sistema económico particular en un momento histórico. El interés público concerniente a la sostenibilidad es, de manera implícita, subyugado al interés privado, sin relevancia alguna de otras consideraciones de riqueza no instrumentales, que son importantes para el funcionamiento de un sistema económico. Un momento histórico con desafíos institucionales en economías en desarrollo con fuentes insustituibles de riqueza no material requieren de competencias específicas de comprensión y solución de problemas que parten del cuestionamiento ético, en términos de la relevancia relativa del interés público frente al interés privado. Los desarrollos de la economía institucional así lo demuestran al señalar al interés público como prioritario en la solución de dilemas del prisionero, concernientes al diseño de instituciones sostenibles en una democracia fundada en el goce efectivo de derechos.

No es difícil imaginar el tipo de presión que originan los conflictos entre los intereses públicos y privados que subyacen en la aplicación de la profesión, tanto en el campo de la política económica como en el campo científico. Existe un conflicto de intereses latente en las definiciones propuestas y la percepción de la sociedad que determina el prestigio y la credibilidad de la profesión. La tensión interna surge como consecuencia de una falta de definición de lo que se considera como interés público, y lo que significa servir al interés público en un marco de reflexión ética, inmerso al mismo tiempo en las actividades propias del economista, relacionadas con la comprensión de los procesos sociales $\mathrm{y}$ económicos que definen lo que se produce, cómo se acumula y cómo se distribuye lo que se considera riqueza con valor económico en una sociedad. La riqueza fundada en la prioridad del interés público sobre el interés privado tendrá un matiz distinto, al menos en lo que atañe al diseño de instituciones relevantes para el desarrollo humano.

De acuerdo con lo anterior, las definiciones propuestas no concuerdan con las actuales exigencias de la profesión y requieren de una redefinición acorde a la evolución histórica de la filosofía de la ciencia económica. Teniendo en cuenta que el objeto de la economía 
se revalida frente al interés público en una economía de mercado, la metodología que subyace al sujeto que realiza el estudio requiere de un nuevo planteamiento para facilitar la incorporación de procedimientos en la profesión afines al interés público.

La crisis económica mundial del 2008, originada en los mercados financieros, reveló la creciente inoperancia de la teoría económica dominante para anticipar y evaluar los efectos perjudiciales del colapso financiero. El confinamiento del análisis económico a un análisis de equilibrio intensifica aún más las tensiones con la opinión pública, pues resulta difícil sostener la hipótesis de mercados eficientes y de expectativas racionales al replicar comportamientos humanos que se acercan más al riesgo moral y la selección adversa que al agente representativo que conoce y respeta las reglas del mercado. Los modelos matemáticos de consistencia interna, ampliamente utilizados en el análisis económico, carecen de consistencia externa y aunque constituyen estructuras lógicas ensambladas bajo la rigurosidad de la demostración matemática, describen el comportamiento real de los mercados financieros. Las demostraciones no son un sustituto perfecto de la contrastación empírica. Esta observación radical permite plantear la hipótesis central de discusión concerniente a la prioridad implícita en el ejercicio profesional del economista en términos del interés privado sobre el interés público, al asumir una interpretación metodológica en el momento de resolver un dilema ético. Es necesario entonces contemplar una ética social fundada incluso en los recientes avances de la teoría económica en lo que atañe a la importancia del interés público para la sostenibilidad de instituciones que median el acto económico cuando de resolver dilemas éticos se trata.

Los modelos económicos de la teoría económica dominante desacreditaron la profesión como teoría de crisis $\mathrm{y}$ en cuanto a su deber público, por dos razones; primero, al considerarse como la única explicación fiable del comportamiento económico en condiciones de incertidumbre, y segundo, al fallar en la transmisión veraz de las limitaciones y fortalezas de los modelos económicos. Esto lo describen autores como Galbraith (2010), cuando presenta el reporte de la crisis financiera al Congreso de los Estados Unidos; Stiglitz (2008), quien describe la economía como ciencia desacreditada; Buiter (2009), cuando comenta la desafortunada inutilidad de la economía monetaria de frontera; Kaletsky (2009), cuando esgrime la responsabilidad de los economistas en la crisis financiera; y Colander et al. (2009), cuando sostienen que la raíz de la falla sistemática de la profesión radica en la insistencia de elaborar modelos que por construcción desconocen elementos acerca de la formación de expectativas de los agentes económicos que, comportándose en 
redes de información, distancian el agente representativo neoclásico como arquetipo de la realidad económica.

La inoperancia de la teoría económica dominante según Krugman (2009), obedece a la confusión sistemática entre belleza, definida como consistencia interna de modelos matemáticos, y verdad, definida como el comportamiento observado de los individuos en el mercado financiero. En su opinión, ni la teoría neoclásica, ni la teoría neokeynesiana pudieron haber pronosticado con anticipación el colapso financiero que ocasionó la mayor crisis económica desde la gran depresión de 1929, debido a que ambas vertientes aceptan la hipótesis de mercados eficientes y expectativas racionales. Lo que parece indicar que la ciencia económica regida por la doctrina dominante se aproxima más a un ejercicio de persuasión forzosa que a un ejercicio de búsqueda de una verdad científica bajo un enfoque de ética social. Al respecto, McCloskey presenta una definición de ciencia que permite aclarar la disyuntiva, al referirse a la constante omisión por parte de los economistas a discutir temas éticos, "The world, [...] "science", needs a careful definition if it is not to obstruct thinking; it means anciently "the study of the available means of unforced persuasion" not 'lying bloviation"” (2009, p. 5).
Con lo anterior no se pretende argumentar que la doctrina de pensamiento neoclásica haya mentido por conocimiento a priori, sino como consecuencia del reemplazo de la observación directa de la realidad en la cual ocurre el acto económico por la consistencia matemática de los modelos económicos. En opinión de Freeman (2009), parece un abuso de la lógica al desconocer las preguntas científicas que subyacen a la elección de un marco conceptual a partir del cual se construye una teoría:

(a) What really is this underlying conceptual structure? What presuppositions, what axioms, must we adopt, in order to think in such a way? This is the true application of logic.

(b) What relation does this conceptual structure have to reality? Does it explain the observed facts which any educated person can verify: does it make sense of the world? This is the true application of science.

(c) What relation does it have to the known work of any writers whose theories we are assessing? Does it make sense of their ideas and are we justified, therefore, in attributing this theory to them when we judge their work? This is the true application of honest debate.

All three of these are jointly required for a scientific endeavor. (1999, p. 28) 
La lógica de las ideas no concuerda con los hechos de las transacciones económicas, lo que genera una falla sistemática en las explicaciones no solo de la más reciente crisis financiera, sino como la plantea Krugman (2009),

It is hard to argue that this transformation in the profession was driven by events. True, the memory of 1929 was gradually receding, but there continued to be bull markets, with widespread tales of speculative excess, followed by bear markets. In 1974-4, for example, stocks lost 48 percent of their value. And the 1987 stock crash, in which the Dow plunged nearly 23 percent in a day for no clear reason, should have raised at least a few doubts about market rationality. $(2009$, p. 6$)$

La falla sistemática de la profesión para explicar y anticipar las crisis financieras no es nueva, y de hecho puede tener un origen institucional. La formulación y el avance de la teoría económica bajo la batuta de la teoría ortodoxa dominante han generado procesos de selección adversa académica y captura regulatoria. La selección adversa ocurre como consecuencia de la falta de un criterio de validación externo a la profesión. La ausencia de una estructura regulatoria que realice la verificación de las teorías que se elaboran, disminuye las advertencias necesarias para prevenir la consoli- dación de una teoría incorrecta como correcta. Los referees académicos realizan más un ejercicio de aprobación que un ejercicio de mérito científico. Freeman (2009) describe el proceso mediante el cual a aquellos que están en desacuerdo se les asigna un puntaje de baja calidad, mientras que a aquellos que comparten la postura teórica se les asigna un puntaje de alta calidad. Por lo tanto, un efecto de retroalimentación se consolida seleccionando teoría y educando referees de manera que el único criterio de aprobación sea la ortodoxia. Dicho mecanismo no tiene la posibilidad de eliminar una teoría incorrecta y, aún más preocupante, limita la posibilidad de generar una teoría correcta.

La captura regulatoria ocurre a consecuencia de la existencia de la economía como un sistema regulatorio y autovalidor per se. El consenso que genera la política internacional de organismos multilaterales como el Banco Mundial y el Fondo Monetario Internacional sugiere una importancia relativa de hipótesis como la de mercados eficientes en países de desarrollo. Debido a que las decisiones económicas tienen implicaciones significativas sobre la riqueza de dos de los más importantes agentes económicos en la actualidad, el sistema financiero y los gobiernos, la adopción de una regulación económica genera captura regulatoria al convertir una teoría económica en dogma financiero y político. 
De lo anterior se deduce la necesidad de una reconsideración del marco referencial científico, que rige la construcción de la teoría económica con una ética social sobre la cual el interés público sobresalga al mismo nivel que lo hace el interés privado, otorgando criterios al economista para resolver la tensión entre el interés público y el interés privado. En particular, Freeman (1999 y 2009) propone la incorporación del principio de pluralismo crítico como criterio epistemológico en la ciencia económica. En el pluralismo crítico, el test definitivo de una teoría es la evidencia empírica, que impone una discusión rigurosa entre diferentes alternativas de aproximación a la realidad, que facilita la representación y la comprensión de un enfoque diferente al que se profesa, pero, a la vez, señala una alternativa mucho más productiva para la comprensión precisa de lo que se profesa. El pluralismo crítico asume el entendimiento de cada teoría disponible de un conjunto de teorías alternativas en términos de su relevancia empírica, garantizando consistencia con la realidad que se intenta explicar. La crisis financiera es evidencia prima facie para promulgar la inclusión del pluralismo critico dentro del marco referencial de la profesión, si se con- sidera el deber público del economista ante la sociedad sobre la base de una ética social.

\section{METODOLOGÍA}

Para evaluar el estado actual de la regulación de la profesión en cuanto a la disyuntiva científica planteada por la crisis económica de 2008, se implementó una metodología que combina métodos deductivos, analíticos y comparativos de estudio de casos para una muestra de diez países, ocho países de América Latina, más Estados Unidos e Inglaterra. La muestra fue seleccionada por conveniencia, al reconocer la necesidad de un nuevo modelo de pensamiento económico apropiado a los desafíos de desarrollo económico de las economías de América Latina. Al mismo tiempo se complementó el análisis con la evaluación de códigos de ética existentes, haciendo uso de los resultados del proyecto RES$\mathrm{PECT}^{8}$, que consolida las principales recomendaciones de 40 códigos de ética alrededor del mundo, al igual que el código de ética para la profesión en países como Colombia, Puerto Rico, Venezuela, Ecuador, Costa Rica, México, España y Argentina (Herman \& Flecker, 2003).

\footnotetext{
${ }^{8}$ El proyecto RESPECT del Programa de Tecnologías de la Sociedad de la Información, de la Comisión Europea, propone directrices profesionales y éticas para la realización de la investigación socioeconómica, promoviendo el respeto por la ética de la investigación, la propiedad intelectual, la confidencialidad, a la cualificación de profesionales, a las normas profesionales, y a los usuarios de las investigaciones, sus objetivos se centran en desarrollar estándares para el análisis de la investigación europea y trasnacional de investigación socioeconómica en especial relacionadas con la sociedad de la información, promoviendo principios de ética y buscando consolidar políticas de calidad (ver más en http://www.respectproject.org/main/aims.php).
} 


\section{RESULTADOS}

La reseña de ocho países latinoamericanos, más Estados Unidos e Inglaterra presenta ausencia absoluta y relativa en cuanto a la existencia de lo que podríamos sugerir se ha convertido en un principio ético: el pluralismo crítico. La Tabla 1 presenta la información más relevante del tamaño y la estructura de la regulación. Una regulación promedio consta de 35 artículos que se distribuyen en cinco segmentos: disposiciones generales en torno al ejercicio de la profesión, de los organismos profesionales, de las sanciones $\mathrm{y}$ recursos, y de las disposiciones transitorias. No todos los segmentos se encuentran presentes en todos los casos, pero todos sin excepción discuten la relevancia del ejercicio de la profesión sin hacer referencia directa o indirecta al pluralismo crítico.

Entre las reformas más recientes se encuentran las de Ecuador, y Costa Rica de 1998, mientras que las que cuentan con mayor experiencia en su

Tabla 1. Tamaño y estructura de la regulación sobre la profesión

\begin{tabular}{|c|c|c|c|c|c|c|c|c|c|}
\hline \multirow[b]{2}{*}{ País } & \multirow{2}{*}{ 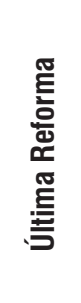 } & \multicolumn{3}{|c|}{$\begin{array}{l}\text { Tamaño de la } \\
\text { Regulación }\end{array}$} & \multicolumn{5}{|c|}{ Estructura de la regulación } \\
\hline & & Tít. & Cap. & Art. & 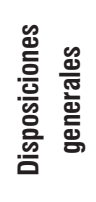 & 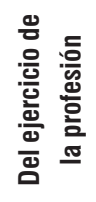 & 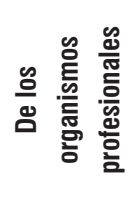 & 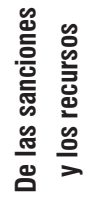 & 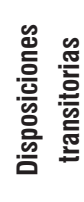 \\
\hline Colombia & 1990 & 2 & 0 & 29 & No & Si & $\mathrm{Si}$ & $\mathrm{Si}$ & No \\
\hline Méjico & 1993 & 0 & 8 & 73 & $\mathrm{Si}$ & $\mathrm{Si}$ & $\mathrm{Si}$ & $\mathrm{Si}$ & $\mathrm{Si}$ \\
\hline Perú & 1986 & 0 & 0 & 21 & No & $\mathrm{Si}$ & $\mathrm{Si}$ & No & No \\
\hline Venezuela & 1971 & 0 & 4 & 49 & $\mathrm{Si}$ & $\mathrm{Si}$ & $\mathrm{Si}$ & $\mathrm{Si}$ & $\mathrm{Si}$ \\
\hline España & 1977 & 5 & 0 & 16 & $\mathrm{Si}$ & $\mathrm{Si}$ & No & No & No \\
\hline Argentina & 1973 & 2 & 0 & 28 & No & $\mathrm{Si}$ & Si & No & No \\
\hline Ecuador & 1998 & 0 & 4 & 11 & $\mathrm{Si}$ & $\mathrm{Si}$ & $\mathrm{Si}$ & No & $\mathrm{Si}$ \\
\hline Costa Rica & 1998 & 0 & 10 & 57 & $\mathrm{Si}$ & $\mathrm{Si}$ & $\mathrm{Si}$ & $\mathrm{Si}$ & $\mathrm{Si}$ \\
\hline
\end{tabular}

Fuente: elaboración de los autores con base en la regulación de la profesión en los 10 países que componen la muestra de estudio. 
implementación ocurren en Argentina y Venezuela, las cuales datan de 1873 y 1971 respectivamente. En todos los casos se observa una aproximación fundada en la definición de la profesión planteada por la Organización Internacional del Trabajo al definir las tareas de un economista, a excepción del caso de México, por tratarse de una Ley General de las Profesiones. De igual manera, las disposiciones generales aducen a requerimientos formales como la obtención de título profesional, mientras las sanciones sugieren las contravenciones legales en que incurre la práctica profesional no debidamente calificada por una universidad u organismos profesionales. Solamente en el caso del estatuto profesional de economistas de España no se menciona en forma directa la formación de organismos profesionales que emiten certificados para ejercer la profesión, lo que indica un mayor nivel de competencia que las demás regulaciones. En los casos de Estados Unidos e Inglaterra se presenta la definición de la profesión propuesta por el Bureau of Labor Statistics, y el Department for Work and Pensions respectivamente.

El segmento dentro de la estructura de las reformas de mayor interés (para los propósitos del presente documento) es el concerniente al ejercicio de la profesión, en particular a las actividades distintivas que cada regulación asocia a la profesión. En este caso, la regulación hace especial énfasis en las técnicas de la profesión como capacidad de llevar a cabo análisis económicos utilizando procedimientos previamente establecidos y aceptados dentro del quehacer aplicado de la profesión. Como se discutió anteriormente, la política económica en el ámbito de la técnica debe incorporar una reflexión ética con el propósito de solventar la tensión del interés privado-público. En este caso, el principio ético de pluralismo critico que cita a la honestidad en la construcción de la ciencia, es remplazado por un principio de responsabilidad, en cuanto a la utilización de técnicas en el manejo y el procesamiento de la información.

Sin un acuerdo tácito de voluntades, que sugiere un marco referencial para la reflexión ética, el profesional se enfrenta a la disyuntiva de distinguir prácticas responsables de prácticas irresponsables, sin una guía profesional en cuanto a la utilización de información, su validación estadística y econométrica. Mayer (2009) presenta una discusión que puede servir de guía constructiva acerca de los procedimientos que atentan contra la integridad y la honestidad de la profesión.

Las normas que regulan la profesión del economista en los países que componen la muestra seleccionada no hacen alusión directa o indirecta a los principios de pluralidad crítica y responsabilidad, descritos anteriormente; 
lo que genera un vacío en la práctica orientada a una dedicación con esmero por el mejoramiento de la profesión en tanto expresión creativa científica, es decir, no son baluartes que promuevan y promocionen el arte de la economía en la representación de la realidad con fines de interpretación, ni representan soluciones al dilema del ejercicio de la profesión y su impacto en el interés público.

Se hace necesario recurrir a acuerdos informales que promuevan una mejor práctica basada en conductas estrictamente éticas. Dentro de dichos acuer- dos informales sobresalen las propuestas de las asociaciones profesionales como el proyecto RESPECT en Europa, el código de ética de la Asociación Nacional de economistas Forenses de los Estados Unidos, el código de ética de la Asociación de Economistas de Puerto Rico, el código de ética Profesional del Economista en Colombia, y la propuesta académica de un código de ética para los economistas sugerido por De Martino (2005). La Tabla 2 presenta un contraste de los principios éticos que propone cada código. Vale la pena resaltar que el código de ética propuesto por Herman y Flecker

Tabla 2. Códigos de ética

\begin{tabular}{|l|l|l|l|}
\hline \multicolumn{1}{|c|}{ Fuente } & \multicolumn{1}{|c|}{ País } & \multicolumn{1}{c|}{ Autor } & \multicolumn{1}{c|}{ Principios y normas éticas } \\
\hline $\begin{array}{l}\text { Code of Professional } \\
\text { Conduct in Socio- } \\
\text { economic Research, } \\
\text { and Data Base of Pro- } \\
\text { fessional Bodies }\end{array}$ & $\begin{array}{l}\text { Australia, Cana- } \\
\text { dá, Sudáfrica, } \\
\text { Estados Unidos } \\
\text { y Europa }\end{array}$ & $\begin{array}{l}\text { The Institute for } \\
\text { Em ploy m n nt } \\
\text { Studies. } \\
\text { Respect Proj- } \\
\text { ect. 2004. }\end{array}$ & $\begin{array}{l}\text { Integridad, transparencia, eficiencia, po- } \\
\text { sición crítica frente a clientes, agradeci- } \\
\text { mientos, referencias, plagio, protección } \\
\text { de datos, interacción con colegas, inte- } \\
\text { racción con clientes, y contratistas, de- } \\
\text { claración de financiamiento, evaluación } \\
\text { científica, conducta profesional. }\end{array}$ \\
\hline $\begin{array}{l}\text { National Association } \\
\text { for Forensic Econom- } \\
\text { ics }\end{array}$ & Estados Unidos & NAFE, 2004. & $\begin{array}{l}\text { Contratación, compensación, diligencia, } \\
\text { declaración, consistencia, conocimien- } \\
\text { to, referencias, y responsabilidad. }\end{array}$ \\
\hline $\begin{array}{l}\text { Asociación de Econo- } \\
\text { mistas de Puerto Rico }\end{array}$ & Puerto Rico & AEPR, 2007 & $\begin{array}{l}\text { Independencia, legalidad, integridad, } \\
\text { confidencialidad, formación, perfeccio- } \\
\text { namiento, legalidad. }\end{array}$ \\
\hline $\begin{array}{l}\text { A Professional Ethics } \\
\text { Code for Economists }\end{array}$ & Estados Unidos & $\begin{array}{l}\text { D o c u m e n t o } \\
\text { científico }\end{array}$ & $\begin{array}{l}\text { Primun non nocere, principio de pre- } \\
\text { caución. }\end{array}$ \\
\hline
\end{tabular}

Fuente: elaboración de los autores con base en la regulación de la profesión en los 10 países que componen la muestra de estudio. 
(2003) en el marco del Respect Project, del Instituto de Estudios de Empleo, es el resultado de la compaginación de 40 códigos de ética alrededor del mundo que, aunque está orientado a la investigación socioeconómica, la cual incluye a la economía, permite establecer principios comunes a prácticas aplicadas de política económica y publica de la profesión. En dicho código se destacan los principios de integridad, definido como la obligación del profesional de mantener una actitud sin sesgos y de mente abierta en cuanto a la formulación y selección de preguntas y métodos de investigación, al igual que el principio de transparencia, que se refiere a la posibilidad de error inmersa en la investigación social, como justificación para la disponibilidad de la información concerniente a las elecciones realizadas por el profesional en el ejercicio de sus facultades. Así mismo sobresale el principio de eficiencia, asociado al compromiso del investigador de presentar la mejor alternativa costo-beneficio para el contratista.

El código de ética de la National Association of Forensic Economics (2004), (NAFE por sus siglas en inglés) describe la conducta profesional en cuanto es requerida como evidencia en ámbitos judiciales. Los principios de contratación, compensación y diligencia hacen referencia al establecimiento de contratos, tarifas y utilización de métodos, mientras que los principios de declaración, consistencia, conocimiento, referencias y responsabilidad comportan el manejo de información detallada del caso judicial en cuestión, y el manejo de la consistencia de los argumentos, independientemente de que se encuentre del lado del defendido o del lado del acusado. En todos los casos, la posición de la práctica profesional está supeditada al conocimiento actualizado que debe poseer cada profesional, al derecho de no referenciar las fuentes de su ejercicio profesional y a la responsabilidad de conocer y aplicar el código de ética.

En el código de ética de la Asociación de Economistas de Puerto Rico (2007) se elabora a partir de seis principios éticos, la independencia intelectual y moral del economista, que se convierte en garantía de los intereses de los destinatarios en términos de una práctica profesional objetiva. Tales principios señalan la legalidad concerniente a una práctica profesional, en la cual el economista siempre actuará conforme al ordenamiento jurídico, a la integridad, que indica honestidad, lealtad y veracidad en el desempeño de las labores profesionales, a la confidencialidad, en la cual se esgrime el derecho que tiene el economista a no revelar información confidencial concerniente a su trabajo profesional, a la formación y perfeccionamiento que aduce a la continua actualización de 
los conocimientos como obligación del economista, y a la lealtad, que hace referencia a la actitud respetuosa, leal y solidaria con sus colegas, compañeros y en sus relaciones profesionales, como aspectos explícitos sobre los cuales la práctica profesional puede resolver la tensión del interés privado sobre el interés público.

\section{DISCUSIÓN}

En Colombia, el código de ética profesional del economista hace especial énfasis en anteponer los valores de la nacionalidad y los intereses de la patria y la sociedad a cualquier otro. Sin embargo, no existe consenso acerca de lo que significa interés por la patria y la sociedad. Si resulta difícil distinguir un interés público en un mercado en particular, resultará en consecuencia mucho más difícil distinguirlo en toda la economía. Para expresar detalladamente unos principios éticos más concretos y de fácil aplicación, De Martino (2009) sugiere una alternativa del Óptimo de Pareto que podría ser considerado por muchos economistas como la versión aplicada del principio que antepone los intereses de la patria a cualquier otro.

Siguiendo a De Martino (2009), el criterio de Óptimo de Pareto se encuentra en el núcleo del modelo de eficiencia que argumenta que a través del intercambio comercial es posible alcanzar una distribución de los recursos, de tal forma que no es posible mejorar la utilidad de un individuo o grupo de individuos sin empeorar la utilidad de otro individuo o grupo de individuos. La formulación de política económica en condiciones de incertidumbre en las cuales no existe separación entre el sujeto, en este caso el profesional, y el objeto, la sociedad, elige de todos los posibles resultados aquellos que cumplen con la condición de optimalidad en forma estricta y potencial. Sin embargo, en condiciones de incertidumbre con separación de sujeto y objeto ocasionado por la presencia de ideologías o interés particulares aparece un riesgo de afectar involuntariamente a un individuo o grupo de individuos, aun en condiciones de eficiencia. La prueba de que dicho argumento falla como indicador de los intereses de la patria es la imposibilidad de garantizar que ningún segmento de la población resultará perjudicado antes y después de la implementación de una política económica.

El principio sugerido por De Martino (2009) es primun non nocere (primero no herir), que emerge como una consideración más concreta en ámbitos en los cuales existe una separación entre sujeto y objeto en condiciones de incertidumbre. El principio se deriva del hecho de que en condiciones de incertidumbre es imposible prever con seguridad los efectos de la política sobre el bienestar de toda la población y, por lo tanto, el economista debe obrar con 
precaución en el diseño e implementación de política económica.

La defensa del neoliberalismo, como lo presenta De Martino (2009), por parte de los economistas, es una conducta antiética dado que, en condiciones de incertidumbre con separación de objeto y sujeto, las intervenciones de política económica tienen el potencial de perjudicar la situación económica de grupos de individuos en la sociedad, y, por lo tanto, su implementación y discusión debe realizarse bajo el principio de la precaución.

Sin embargo, ni los códigos de ética, ni los acuerdos de ley han facilitado la implementación de principios de pluralismo crítico, ni de precaución en el diseño de política económica. Los códigos de ética son considerados acuerdos voluntarios entre profesionales no regidos por ley, a excepción del caso de Colombia en donde existe un decreto que regula la conducta profesional. Por otro lado, las leyes que reglamentan el ejercicio de la profesión no representan articulaciones adecuadas para resolver las tensiones que ocurren en la relación sujeto y profesión en cuanto a la disyuntiva de interés público e interés privado, en un entorno de incertidumbre. Por lo tanto, los poderes regulatorios que define la economía sobre la producción, la distribución y el consumo de bienes para la satisfacción de necesidades, pueden ser manipulados para la obtención de beneficios privados en contra del interés público.

La regulación es necesaria en la medida en que disminuye la información asimétrica que afecta la transacción implícita que ocurre entre un principal, la sociedad y un agente, el economista. La información asimétrica en tal sentido, tiene dos consecuencias: (1) selección adversa en cuanto a la calidad de alternativas de política implementadas, y (2) riesgo moral, en cuanto a la integridad y honestidad del profesionalismo del economista de servir al interés público.

Un economista que se desempeña en un cargo de interés público puede responder a la tensión entre el interés público y el interés privado modificando la relación de intercambio entre las decisiones económicas y las decisiones éticas, prevaleciendo en condiciones de incertidumbre el criterio económico no pluralista de imposición por persuasión forzada, al criterio por persuasión científica no forzada. La falta de pluralismo crítico y de responsabilidad puede conducir, por tanto, a la disminución de la calidad promedio del debate político en cuanto a alternativas económicas, y favorecer la construcción de un dogma ideológico a través de la implementación de políticas públicas elegidas sobre un criterio de persuasión y no de un mérito o relevancia empírica. 
La selección adversa ocurre como consecuencia de la incapacidad de la sociedad de diferenciar las alternativas de políticas de alta calidad de las alternativas de baja calidad. En una sociedad democrática, los individuos expresan dicha incapacidad reduciendo el valor relativo que están dispuestos a sacrificar por obtener cada política económica. Ejemplos de dicha reducción en el precio relativo es una baja participación electoral o una alta evasión de impuestos. Si el costo de mantener alternativas de alta calidad es mayor a la reducción del precio relativo, las alternativas de alta calidad son eliminadas, reduciendo la calidad promedio de las alternativas disponibles, lo que a su vez genera la repetición del proceso, empeorando la calidad del desempeño de la práctica profesional en cuanto al interés público, y desplazando a las políticas económicas de mayor calidad.

El riesgo moral ocurre una vez el economista se encuentra en una posición de interés público. Debido a la existencia de asimetría de información, la sociedad no puede identificar con precisión la calidad del servicio en términos de pluralidad y contrastación empírica. Una vez contratados, el incentivo para mantener alternativas distintas cede más a la presión de referees o principales que al interés público, y la calidad del desempeño en función del interés público disminuye. Por ejemplo, si la contratación ocu- rre por etapas, una etapa de diagnóstico y otra de implementación, existe la posibilidad de separar principales e incorporar controles a la presión de grupo, permitiendo un espacio plural y crítico. Sin embargo, cuando las dos etapas ocurren como parte del mismo servicio profesional, el profesional no solo tiene el incentivo de eliminar la autocrítica que busca articular en forma más conveniente el interés público con el interés privado, sino que aparece un incentivo de captura regulatoria para inducir la demanda de servicios no requeridos.

Las fallas de mercado atribuibles al ejercicio del economista en un entorno de incertidumbre y sin separación del objeto y el sujeto de la transacción incluyen problemas de presión de grupo académico, selección adversa, riesgo moral, captura regulatoria de un bien público a través de organizaciones buscadoras de renta que surgen en el interior de las organizaciones públicas. La provisión en cada caso de políticas económicas con serios desarreglos institucionales en torno a los principios de pluralismo crítico, responsabilidad, primun non nocere y precaución, afecta en consecuencia el bienestar de la sociedad y justifica una regulación pública.

Como lo describen Stephen, Ehlermann y Atanasiu (2006), en los mercados en los cuales los consumidores no son frecuentes y no existe formación de reputación a través de redes 
de transmisión de información de consumidores más experimentados, el gobierno debe proveer la información necesaria para incrementar el bienestar de los consumidores. Los consumidores frecuentes de servicios profesionales obtienen información adicional sobre la calidad del servicio y reducen la posibilidad de ocurrencia de una falla de mercado, al mismo tiempo que generan competencia entre oferentes como consecuencia de la información adquirida en repetidas contrataciones. Por ende, la reputación reduce los problemas de información asimétrica al transmitir información entre consumidores frecuentes y consumidores poco frecuentes.

Existen arreglos institucionales que corrigen la falla de mercado evitando la formación de carteles o monopolios. Garoupa (2004) presenta tres tipos de regulación que surgen como alternativas:

1. Regulación de entidades públicas.

2. Autoevaluación como sinónimo de regulación efectiva de la práctica profesional.

3. Regulación a través de entidades privadas.

Dentro de la regulación implementada por las entidades públicas sobresale la regulación a los programas académicos de economía, la certificación para el desarrollo de actividades profesionales, y la emisión de licencias.

La autorregulación promueve una mayor asociación al emitir licencias profesionales, sin embargo, según Garoupa (2004), tal alternativa soluciona parcialmente el problema de información asimétrica, debido a que ni la asociación de profesionales, ni el gobierno tienen un mejor conocimiento acerca de la calidad de los servicios profesionales. Además, es mucho menos flexible a la alternativa de generar incentivos directamente al profesional, lo cual genera costos adicionales que debe asumir el gobierno y no el profesional, quien es el que mejor conoce su desempeño profesional.

Las asociaciones de profesionales tienen mayor información acerca de la manera como se desenvuelve la práctica profesional en relación con la información del público en general, que aun después de haber experimentado la política, desconoce si se trata de un servicio de alta o baja calidad. Sin embargo, las asociaciones profesionales generan monopolios que derivan en un incremento en los costos asociados a la implementación de los servicios profesionales. La regulación de entidades privadas sin ánimo de lucro plantea algunas alternativas a los problemas de información asimétrica. Establecer agencias independientes de consumidores frecuentes puede 
permitir una transferencia de información de mercado entre agentes, favoreciendo la contratación y el prestigio de la profesión en cuanto al servicio del interés público.

Algunos instrumentos de regulación utilizados son restricciones a la entrada con precaución a la formación de carteles, restricciones a la publicidad para promover competencias al interior de la profesión, restricciones en tarifas y honorarios, restricciones sobre la forma organizativa y sobre la conducta y los procedimientos. Las restricciones a la entrada incrementan la calidad de los servicios profesionales, pero disminuyen la competencia creando monopolios. Las restricciones usualmente se ejecutan en tres niveles según Garoupa (2004):

1. Modificando los contenidos programáticos de la instrucción.

2. Implementando exámenes al finalizar la preparación académica.

3. Imponiendo normas de certificación a las instituciones que emiten el conocimiento correspondiente.

No obstante, como lo plantea Stephen (2006), la regulación concerniente a la entrada al ejercicio de la profesión es un tópico más elaborado debido a la existencia de externalidades. En cualquier caso, la restricción a la entrada no puede imponerse sobre la base de proteger los intereses económicos de los profesionales agremiados.

Las restricciones sobre la publicidad son permitidas cuando es productiva en el sentido de que permite identificar la calidad del servicio profesional. Como lo describe Stephen (2006), cuando la publicidad en precios es permitida, el precio transmite la información sobre la calidad en forma indirecta y, por lo tanto, la publicidad de precios incrementa el bienestar del consumidor. En oposición a lo anterior, cuando la publicidad de precios es realizada por oferentes de baja calidad, el resultado es el opuesto. La publicidad afecta la calidad del servicio, y la percepción sobre la calidad afecta la demanda del servicio.

Las restricciones sobre tarifas pueden ser vistas como una forma de asegurar una prima de confiabilidad sobre el profesional, quien es el que mejor conoce de la calidad del servicio que ofrece (Garoupa, 2004). No obstante, Stephen (2006) señala cómo las restricciones equivalen a la formación de carteles vía precios. Además, las restricciones sobre las formas organizacionales son difíciles de justificar desde el punto de vista del interés público, de acuerdo con la argumentación planteada por Garoupa (2004). Por otro lado, algunos aspectos de las profesiones pueden favorecer las asociaciones y disminuir el costo en la prestación 
del servicio, empero generan un monopolio que reduce los incentivos a la entrada al mercado y a la innovación en la prestación de los servicios profesionales. Según Stephen (2006), no existe ninguna base conceptual para prohibir la competencia de precios en el mercado de los servicios profesionales. Si es preciso incorporar la separación de la función de agencia de la función de servicio para evitar la demanda inducida, el estándar mínimo de regulación debe imponer otro tipo de instrumentos orientados a favorecer la calidad en la prestación de servicios particulares en mercados particulares.

De otro lado, las restricciones sobre la conducta proponen la introducción de estándares profesionales y de ética en el interior de la profesión, los cuales deben propender a un mayor pluralismo y una mejor definición de lo que es el interés público y lo que significa servir el interés público. Desde el punto de vista de diseño de incentivos, como lo plantea Garoupa (2004), la respuesta de los profesionales será establecer una agenda de costos en cada una de las actividades de definición, monitoreo y provisión del servicio. No obstante, existe el riesgo moral al ser los profesionales quienes establezcan su propia normatividad en cada una de las actividades, lo cual pone en riesgo la calidad del servicio prestado.

En resumen, cada alternativa de instrumentos presenta una normativa par- ticular con un beneficio y costo asociado. Como lo plantea Stephen (2006), la evaluación general señala al mercado de servicios profesionales como una institución sujeta a una competencia de mercado regida por la ley con el objeto de imponer estándares de calidad a manera de una responsabilidad directa de las agremiaciones profesionales. Por otro lado, las agremiaciones profesionales requieren de la incorporación de los principios de pluralismo crítico, responsabilidad, primun non nocere y precaución, como principios éticos en el desempeño profesional de un economista.

\section{CONCLUSIONES}

El actual régimen institucional tanto legislativo como ético de diferentes países en Latinoamérica y el mundo no soluciona la tensión que genera el interés privado y el interés público en el desempeño profesional de un economista en una economía de mercado. Debido a que el ejercicio de un economista ocurre en un entorno de incertidumbre en donde no hay separación entre el objeto y el sujeto de intervención es necesario incorporar los principios de pluralismo crítico, responsabilidad, primun non nocere y precaución en el ejercicio de la profesión. El actual marco de acción no ofrece herramientas a modo de competencias profesionales que le permitan distinguir al profesional la disyuntiva metodología 
del dilema ético. Es necesario, en consecuencia, implementar una ética social en el entrenamiento y en la práctica, sea esta teórica o aplicada de la ciencia económica, en el momento de comprender y brindar alternativas en la construcción de un modelo de pensamiento y práctica profesional, en entornos que acometan la difícil tarea de diseñar institucionalmente economías sobre la base del interés público.

Existen diferentes arreglos institucionales que pueden solucionar los problemas de información asimétrica, los cuales desembocan en una baja calidad media de la práctica profesional y un riesgo moral latente frente a la imposibilidad de resolver un dilema ético, implícito en la práctica profesional. No obstante, todos los arreglos presentan una desventaja en términos de captura regulatoria, debido a que la economía es en sí misma un arreglo institucional factible de ser explotado por buscadores de renta, sobre el fundamento de concepciones ideológicas que priorizan un modelo de pensamiento sobre otro, sin una base empírica reconocida y aceptada bajo un principio de pluralismo crítico.

En cualquier caso, el mercado de servicios profesionales debería estar sujeto a una competencia de mercado regida por la ley para imponer estándares de calidad, los cuales dependen de grandes inversiones controladas y reguladas. Tal regulación atañe a una responsabilidad directa de las agremiaciones profesionales, que tienen el deber moral de dotar a los profesionales de protocolos orientados a disminuir las tensiones del interés público y privado, y a los Estados, instituciones y demás organizaciones privadas para realizar las inversiones que permitan ejercer completamente dichos códigos de ética.

\section{AGRADECIMIENTOS}

Los autores agradecen el apoyo financiero de la Universidad Católica de Colombia en el desarrollo de la investigación. Cualquier responsabilidad es exclusiva de los autores. 


\section{REFERENCIAS}

Allais, M. (1978). La economía como ciencia. En C. Dagum (comp.) Metodología y crítica económica (p. 661). México: Fondo de Cultura Económica.

Asamblea Legislativa de Costa Rica. (1998). Ley Orgánica del Colegio de Profesionales en Ciencias Económicas. Recuperado de http://www.cpcecr.com/ leyesyreglamentos.html.

Asamblea Legislativa de Puerto Rico. (2007). Reglamento y Código Ético, Asociación de Economistas de Puerto Rico. Recuperado de http://www.oslpr. org/v2/documentos.aspx

Bejarano, J. (1999). La profesionalización en economía. En J. Bejarano (comp.) Hacia dónde va la ciencia económica en Colombia. Siete ensayos exploratorios. Bogotá: Colciencias, Tercer Mundo Editores y Universidad Externado de Colombia, primera edición.

Bloom, L. (2004, September-October). Professions and the Public Interest. Contingencies, 30-34. Retrieved from http://www.contingencies.org/septoct04/professions1.pdf

Buiter, W. (2009). The Unfortunate Uselessness of Most "State of the Art" Academic Monetary Economics. Financial Times. Retrieved from http://blogs.ft.com/maverecon/2009/03/the-unfortunate-uselessness-of-most-state-of-the-art-academic-monetary-economics/

Cohen, P. (2007, July 11). In Economics Departments, A growing Will to Debate Fundamental Assumptions. The New York Times, Section B. Retrieved from http://www.nytimes.com/2007/07/11/education/11economics.htm1 ? ex $=1341806400 \&$ en $=$ bf38f9dddc $8 \mathrm{c} 8 \mathrm{ecc} \& \mathrm{ei}=5090 \&$ partner $=$ rssuserland\&emc $=$ rss

Colander, D., Follmer, H., Haas, A., Goldberg, M., Juselius, K, Kirman, A., Lux, T. \& Sloth, B. (2009). The Financial Crisis and the Systemic Failure of Academic Economics. Kiel Institute for the World Economy, working paper (1489). Retrieved from http://ideas.repec.org/p/kie/kieliw/1489.html 
Congreso de la República Argentina. (1973). Ley 20488. Normas referentes al ejercicio de las profesiones relacionadas con las ciencias económicas. Recuperado de http://www.eco.unrc.edu.ar/auditoria/ley-20488-codigo-deetica-rt-7/.

Congreso de la República de Colombia. (1990). Ley 37 de 1990, sobre la reglamentación del ejercicio de la profesión de economista. Recuperado de http://www.conalpe.gov.co/ltindex.htm.

Congreso de la República de Venezuela. (1971). Ley de ejercicio de la profesión de economista. Recuperado de http://www.gobiernoenlinea.ve/legislacion-view/sharedfiles/272.pdf.

Congreso de la República Peruana. (1986). Ley del ejercicio de la profesión de economista. Recuperado de http://www.cept.org.pe/leyaconomis.pdf.

Congreso de los Estados Unidos Mexicanos. (1993). Ley reglamentaria del artículo 5 constitucional, relativo al ejercicio de las profesiones en el Distrito Federal. Recuperado de http://www.diputados.gob.mx/LeyesBiblio/ pdf/208.pdf.

Congreso Nacional de Ecuador. (2004). Ley 102. Ley sustitutiva a la ley de Ejercicio Profesional de los Economistas y Doctores en Ciencias económicas. Recuperado de http://search.yahoo.com/search;_ylt=A0oG76slcAxMd0 YAdF1XNyoA? $\mathrm{p}=\mathrm{Ley}+$ Sustitutiva $+\mathrm{a}+\mathrm{la}+\mathrm{ley}+\mathrm{de}+$ Ejercicio+Profesio$\mathrm{nal}+\mathrm{de}+\mathrm{los}+$ Economistas $+\mathrm{y}+$ Doctores $+\mathrm{en}+$ Ciencias + econ $\% \mathrm{C} 3 \% \mathrm{~B} 3 \mathrm{mi}-$ cas + and + ecuador $\& \mathrm{fr} 2=$ sb-top $\& \mathrm{fr}=\mathrm{yfp}-\mathrm{t}-701 \& \mathrm{sao}=1$.

De Martino, G. (2005). A Professional Ethics Code for Economists. Challenge, 48(4), 88-104.

De Martino, G. (2009). On the Need for Professional Economic Ethics. Challenge, 52(4), 6-15.

Freeman, A. (1999). The Emperor's Tailor: The Economics and the Crash on '98. Munich Personal RePEc Archive, Paper (6712). Retrieved from http:// mpra.ub.uni-muenchen.de/6712/

Freeman, A. (2009). The Economist of Tomorrow: The Case for a Pluralist Subject Benchmark Statement for Economics. International Review of Eco- 
nomics Education, 8(2), 23-40. Retrieved from http://www.economicsnetwork.ac.uk/iree/v8n2/freeman.pdf

Galbraith, J. (2010). James Galbraith on Economic Theory as a Disgraced Profession, ZeroHedge. Retrieved from http:/www.zerohedge.com/article/ james-galbraith-economic-theory-disgraced-profession.

Garoupa, N. (2004). Regulation of Professions in Portugal: A Case Study in Rent-Seeking. In II Conferência sobre Desenvolvimento Económico Português no Espaço Europeu. Retrieved from http://www.bportugal.pt/pt$\mathrm{PT} /$ EstudosEconomicos/Conferencias/Documents/2004DesenvEcon/13 NGaroupa.pdf

Herman, C. \& Flecker, J (2003). Code of Professional Conduct in Socio-Economic Research, and Database of Professional Bodies. Brighton: The Institute for Employment Studies. Retrieved from http://www.respectproject.org/ standards/prof_conduct.pdf

Kaletsky, A. (2009). Economist are the Forgotten Guilty Men. TimesOnline. Retrieved from http://www.timesonline.co.uk/tol/comment/columnists/anatole_kaletsky/article5663091.ece

Krugman, P. (2009). How did Economist get it so Wrong? New York Times Magazine. Retrieved from http://www.nytimes.com/2009/09/06/magazine/06Economic-t.html?_r=1

Mayer, T. (2009) Honesty and Integrity in Economics, Department of Economics UCDavis, Working Paper Series, 09-2. Retrieved from http://www.econ. ucdavis.edu/working_papers/09-2.pdf

McCloskey, D. (2009). Rhetoric Matters. Ethical Standards in a Humanistic Science of Economics. Challenge, 52(4), 25-31. Retrieved from http://www. jstor.org/stable/40722588?seq=1\#page_scan_tab_contents

Ministerio de Educación de Colombia (1977). Decreto 1268 del 6 de junio de 1977, Código de Ética Profesional del Economista. http://www.conalpe. gov.co/ltindex.htm.

National Association of Forensic Economics (2004). Statement of Ethical Principles and Principles of Professional Practice, NAFE. Retrieved from http:// nafe.net/resources/Documents/NAFE_Ethics_2014.pdf 
Ogus, A. (1994). Regulation: Legal Form and Economic Theory. Oxford: Oxford University Press. Retrieved from http://citeseerx.ist.psu.edu/viewdoc/ download?doi=10.1.1.619.5274\&rep=rep1\&type $=$ pdf

OIT. (2008). Clasificación internacional de ocupaciones. Recuperado de http:// www.ilo.org/public/english/bureau/stat/isco/isco88/2441.htm

Presidencia del Gobierno de España. (1977, 28 de abr.). Estatuto Profesional de Economistas y de Profesores y Peritos Mercantiles, de España. Boletín Oficial del Estado. Recuperado de http://www.ecova.es/docs/25_estatuto_profesional.pdf.

Smith, A. (1988). La riqueza de las naciones. Barcelona: Oikos-Tau.

Stiglitz, J. (2008, December 5). The Triumphant Return of John Maynard Keynes, Project Syndicate. Retrieved from http://www.project-syndicate.org/commentary/stiglitz107/English

Stephen, F. (2004). The Market Failure Justification for the Regulation of Professional Service Markets and the Characteristics of Consumers. In C.D. Ehlermann \& I. Atanasiu (eds.), European Competition Law Annual 2004: The Relationship between Competition Law and (Liberal) Professions. Oregon: Hart Publishing, Oxford and Portland.

Stephen, F., Ehlermann, C-D. (Ed.) \& Atanasiu, I. (Ed.) (2006). The Market Failure Justification for the Regulation of Professional Service Markets and the Characteristics of Consumers. In European Competition Law Annual 2004: The Relationship between Competition Law and (Liberal) Professions. Hart Publishing. Retrieved from https://www.research.manchester. ac.uk/portal/en/publications/the-market-failure-justification-for-the-regulation-of-professional-service-markets-and-the-characteristics-of-consumers(2bb7e944-feaa-4315-b1c8-983f44bc42c5).html

Samuel, G. (2000, September). Governing the Professions: Does Self-regulation Equal Self-Interest? Review. Retrieved from http://www.ipa.org.au/library/ Review52-3\%20Governing\%20professions.pdf. 
Anexo 1. Definiciones y tareas asociadas a la profesión

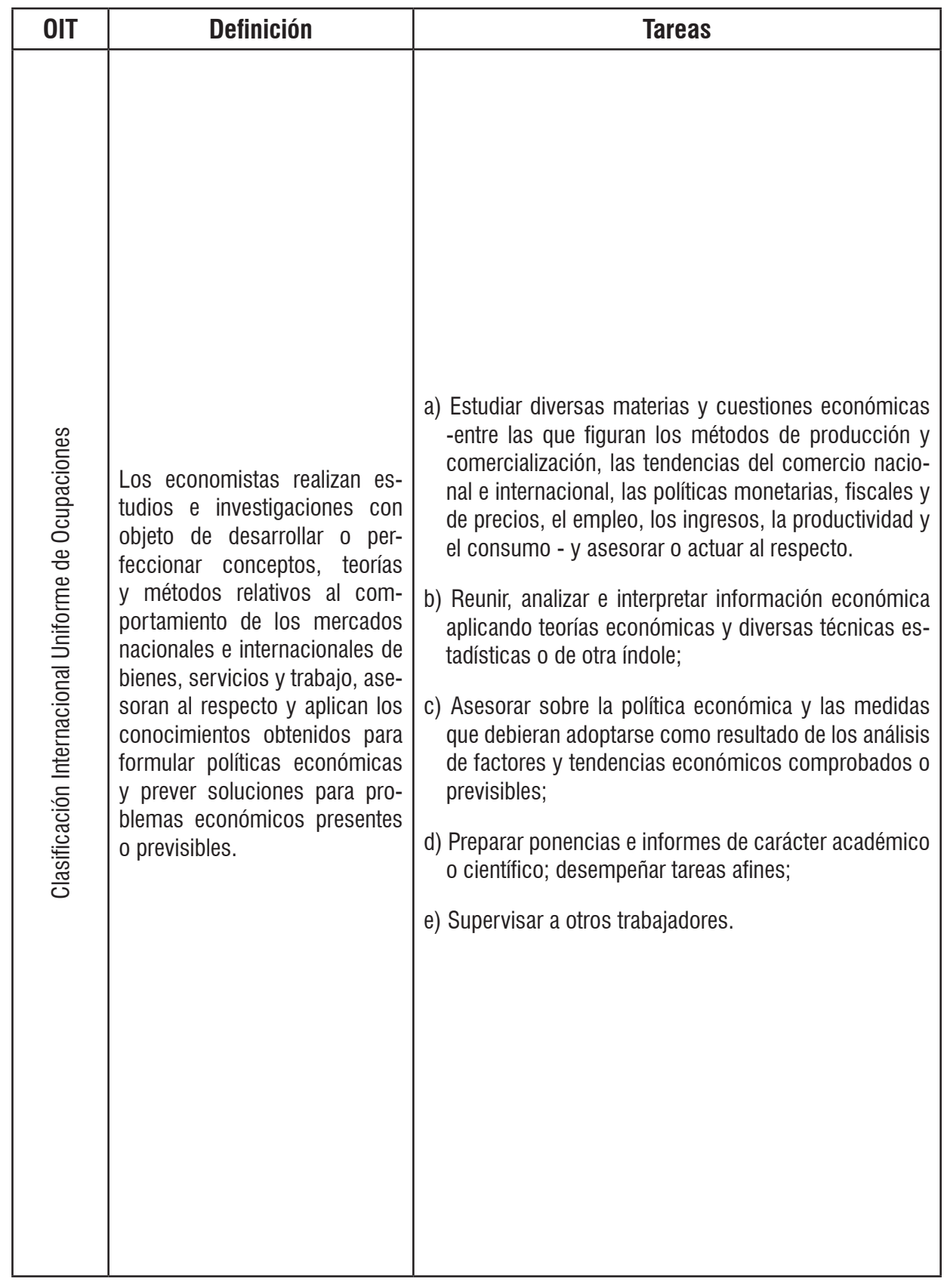




\begin{tabular}{|c|c|c|}
\hline 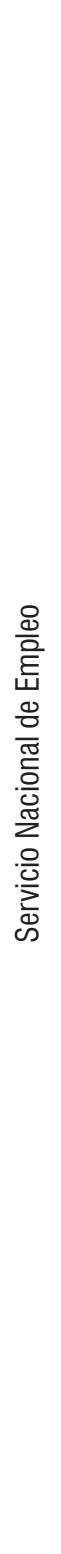 & $\begin{array}{l}\text { Dirigen investigaciones y desa- } \\
\text { rrollan modelos para ilustrar y } \\
\text { pronosticar el comportamiento } \\
\text { económico. Están empleados } \\
\text { por el gobierno y empresas } \\
\text { privadas para asesorar en ma- } \\
\text { terias como finanzas, comercio } \\
\text { internacional, impuestos y mer- } \\
\text { cados agrícolas e industriales. }\end{array}$ & $\begin{array}{l}\text { a) Analizar factores que dminen el crecimiento económi- } \\
\text { co y asesorar a dependencias gubernamentales sobre } \\
\text { políticas para incrementar las actividades económicas. } \\
\text { b) Analizar métodos financieros, costos, técnicas de } \\
\text { producción y políticas de mercadeo para detectar po- } \\
\text { sibles progresos. } \\
\text { c) Analizar problemas relacionados con la actividad eco- } \\
\text { nómica de empresas particulares. } \\
\text { d) Dirigir investigaciones sobre condiciones de merca- } \\
\text { deo en áreas locales, regionales o nacionales para } \\
\text { establecer niveles de ventas y precios para bienes y } \\
\text { servicios y valorar el potencial del mercado y futuras } \\
\text { negociaciones. } \\
\text { e) Dirigir investigaciones y desarrollar modelos para } \\
\text { ilustrar y pronosticar el comportamiento económico } \\
\text { y proyectar métodos para recolección y análisis de } \\
\text { datos. } \\
\text { f) Estudiar el sistema monetario, instrumentos y tipos de } \\
\text { crédito, la operación de los bancos y otras institucio- } \\
\text { nes financieras para desarrollar políticas monetarias y } \\
\text { pronósticos de actividades financieras. } \\
\text { novables y agotamiento de recursos no renovables. } \\
\text { g) Estudiar fórmulas matemáticas y técnicas estadísticas } \\
\text { y aplicarlas a la prueba y cuantificación de teorías y } \\
\text { solución de problemas económicos. } \\
\text { h) Examinar datos estadísticos sobre el intercambio de } \\
\text { bienes y servicios entre naciones. } \\
\text { agrícolas basados en registros de cosechas anterio- } \\
\text { res, mano de obra y condiciones agrícolas y econó- } \\
\text { micas en general. } \\
\text { jonosticar la producción y consumo de recursos re- }\end{array}$ \\
\hline
\end{tabular}

Fuente: elaboración de los autores con base en las definiciones presentadas por la O.I.T. y el S.N.E. de Colombia. 